\title{
The Severity of Hepatitis D in Young Adults of Age 18-25 Years
}

\author{
Zaigham Abbas $^{1}$, Muhammad Ali Qadeer ${ }^{1}$, Haider A. Mandviwalla ${ }^{1}$, Minaam Abbas ${ }^{1,2}$ \\ 1. Gastroenterology and Hepatology, Dr. Ziauddin University Hospital Clifton, Karachi, PAK 2. Gastroenterology and \\ Hepatology, School of Clinical Medicine, University of Cambridge, Cambridge, GBR
}

Corresponding author: Zaigham Abbas, drzabbas@gmail.com

\section{Abstract \\ Background}

Current literature on the prevalence and characteristics of hepatitis D virus (HDV) infection in young adults is limited. This study aims to determine the disease characteristics and severity in young adults.

\section{Methods}

The case records of HDV RNA positive patients of age 18-25 years were analyzed.

\section{Results}

Out of 119 patients, 105 (88\%) patients were male. HBV-DNA was detectable in 83 (70\%). Hepatitis B eantigen (HBeAg) was non-reactive in 99 (83\%). Cirrhosis was identified in 45 (37.8\%) individuals; nine (7.5\%) were classified as Child class B or Child class C. Twenty-four (20.2\%) had a Model For End-Stage Liver Disease (MELD) score of $\geqslant 10$, out of these 16 had a score of 15 or more. The risk of decompensation was calculated according to the Baseline-event-anticipation (BEA) score; eight (6.7\%) patients were at BEA-A (mild risk), 105 (88.2\%) were at BEA-B (moderate risk), and six (5.0\%) were at BEA-C (severe risk). Notable findings in patients with cirrhosis included splenomegaly, low total leucocyte counts, low platelets, high bilirubin, elevated aspartate aminotransferase, gamma-glutamyl transferase and international normalization ratio, low albumin, high AST to Platelet Ratio Index (APRI), and high BEA score. The splenic size, platelet count, and albumin levels were independently associated with cirrhosis $(\mathrm{p}<0.001,<0.001$, and 0.003). A model using a combination of platelet count, albumin, and spleen size was developed to accurately predict cirrhosis in this cohort. It had an area under the receiver operating characteristics (AUROC) of 0.935.

\section{Conclusions}

HDV-infected young adults, age 18-25 years, were at moderate to severe risk of disease progression. About one-third of patients had already developed cirrhosis indicating the aggressive nature of the disease.

Review began 09/29/2020 Review ended 10/03/2020 Published 10/08/2020

\section{○ Copyright 2020}

Abbas et al. This is an open access article distributed under the terms of the Creative Commons Attribution License CC-BY 4.0., which permits unrestricted use, distribution, and reproduction in any medium, provided the original author and source are credited.
Categories: Gastroenterology, Infectious Disease

Keywords: hepatitis delta, hepatitis b, cirrhosis, young adults, statistical model, child class, meld score

\section{Introduction}

Approximately 12 million persons are infected with hepatitis D worldwide [1]. The incidence of hepatitis D virus (HDV) remains high in Asia and is a significant cause of morbidity [2]. In adults, co-infection of hepatitis B virus (HBV) with HDV is usually self-limiting, but can also be associated with a severe form of acute hepatitis and may lead to fulminant hepatitis [3]. Approximately $2 \%$ of patients with co-infection progress to cirrhosis. On the other hand, the superinfection of HDV on HBV eventually leads to chronic HDV infection in $90 \%$ of all cases. This, in turn, results in cirrhosis in $70 \%$ of patients within five to 10 years of the acquisition of the superinfection [4]. The progression to cirrhosis is more rapid, and the incidence of cirrhosis is three times higher with HDV superinfection when compared with HBV mono-infection [5]. Patients with chronic HDV have higher transaminase levels, lower platelet counts and prothrombin activity, and a higher incidence of necroinflammation and significant fibrosis on liver biopsy [6].

HDV infection is an important cause of progressive liver disease in children who are chronic hepatitis B surface antigen (HBsAg) carriers [7]. The acquisition of HDV infection occurs primarily through horizontal transmission [8]. Children infected with HDV typically have minimal symptoms and high levels of HBV replication in the liver with the presence of hepatitis B core antigen ( $\mathrm{HBcAg}$ ) in the liver and hepatitis B eantigen (HBeAg) and HBV DNA in the serum [9]. We previously compared 48 children suffering from hepatitis D with 48 children with hepatitis B monoinfection. Cirrhosis was seen in 13 (27\%) cases with hepatitis D compared with two (4\%) with hepatitis B monoinfection. Six patients with hepatitis D had decompensation at the time of presentation, whereas only one patient with HBV monoinfection had decompensation [10]. 
We, therefore, postulated that if children with HDV infection have a more aggressive form of liver disease than those with HBV monoinfection, patients with HDV in their young adulthood would also display an accelerated course of progression of the disease, with a greater risk of having cirrhosis and decompensation. Existing literature on the prevalence and characteristics of HDV infection in the young adult population between 18-25 years is limited. This study aims to ascertain disease characteristics and severity of hepatitis $\mathrm{D}$ in the young adult population.

\section{Materials And Methods}

A retrospective study of records of HDV RNA positive patients, aged between 18-25 years, who visited our liver clinic, was conducted. Patients with an incomplete profile were excluded. A diagnosis of cirrhosis was made based on ultrasonographic findings. Cirrhosis was considered decompensated in patients that had variceal bleeding, ascites, or hepatic encephalopathy. The risk of decompensation was categorized with the help of baseline-event-anticipation (BEA) Classification [11]. The scoring system is based on male gender = 1 , age $>40=1$, Eastern Mediterranean origin $=1$, platelet count $<100=1$, platelet count $<50=2$, and bilirubin $>$ upper limit of normal $=1$. $\mathrm{BEA}-\mathrm{A}=0-1$ point, $\mathrm{BEA}-\mathrm{B}=2-4$ points, $\mathrm{BEA}-\mathrm{C}=5-7$ points. The Ethics Review Committee reviewed the study protocol and granted access to the data. Our study did not require written/informed consent because of retrospective analysis without disclosing the identity or doing any intervention. The study protocol conforms to the ethical guidelines of the 1975 Declaration of Helsinki as reflected in a priori approval by the institution's human research committee.

All analyses were conducted using the SPSS software (IBM SPSS Statistics Base 26, IBM, Armonk, New York, USA). Categorical variables were compared by Chi-square or Fisher's exact test, as appropriate. For all continuous variables, the mean, standard deviation, median, and range were noted. For comparisons between groups, a non-parametric independent sample Mann-Whitney U test was used, with the significant alpha level (p-value) set at 0.05 . Linear regression analysis was conducted to identify independent factors associated with cirrhosis and receiver operating characteristic (ROC) curves were drawn for these factors. Binomial logistical regression was then used to produce a predictive model combining the three factors and ROC curves for these models were produced.

\section{Results}

A total of 119 patients were included in this study. Most patients were male $(105,88 \%)$, with a median age of 22 years (18-25). 48.7\% (58) of all patients had previously received pegylated interferon (PEG-IFN)-based therapy. Laboratory investigations, clinical parameters, and baseline findings are given in Table 1. 


\section{Cureus}

\section{Clinical parameters}

Gender:male

Age: (years)

Body mass index $\left(\mathrm{kg} / \mathrm{m}^{2}\right)$

Time since hepatitis B diagnosed (months)

Time since hepatitis D diagnosed (months)

Previous PEG-IFN-based therapy Nonresponders Relapsers

Investigations

\author{
HDV-RNA positive \\ HBV-DNA positive \\ HBeAg negative \\ Hemoglobin: (g/dl) \\ Total leucocyte count: $\left(10^{9} / \mathrm{L}\right)$ \\ Platelets: $\left(10^{9} / \mathrm{L}\right)$ \\ International normalization ratio: \\ Bilirubin: (mg/dL) \\ Alanine aminotransferase: (IU/mL) \\ Aspartate aminotransferase: (IU/mL) \\ Gamma glutamyl transferase: (IU/mL) \\ Alkaline phosphatase: (IU/mL) \\ Albumin: (g/dL) \\ Creatinine $(\mathrm{mg} / \mathrm{dL})$ \\ Sodium (mmol/L)
}

HDV RNA quantitative: (log10 IU/mI) (n = 97)

HBV DNA quantitative: (log10 IU/ml) ( $n=73)$ n (\%) or median (range)

$105(88 \%)$

$22(18-25)$

$21.2(15-33.9)$

$36(<1-240)$

$13(<1-168)$

$58(48.7 \%) 46(38.6 \%) 12(10.1 \%)$

$119(100 \%)$

$83(70 \%)$

$99(83 \%)$

5.67 (1.94-8.83)

$3.12(1.00-8.00)$

13.9 (7.4-16.7)

$6.0(2.4-15.4)$

190 (26-443)

$1.01(0.8-2.5)$

$0.7(0.2-10.5)$

69 (25-723)

$54(22-470)$

$40(10-942)$

$4.1(1.8-4.8)$

$0.8(0.3-1.9)$

139 (121-144)

TABLE 1: Clinical characteristics and laboratory investigations of the study patients

PEG-IFN: Pegylated interferon; HDV: Hepatitis D virus; HBV: Hepatitis B virus; HBeAg: Hepatitis B e-antigen.

HBV-DNA was detectable in 83 (70\%). HBeAg was non-reactive in 99 (83\%). This study only included chronic hepatitis D patients with detectable HDV RNA (qualitative). Out of these, 97 had a quantitative HDV RNA test as well. The median HDV RNA level in this latter group was $5.67 \log 10 \mathrm{IU} / \mathrm{ml}$.

Forty-nine (41.2\%) patients had an APRI score of more than one. Cirrhosis was identified in 45 of these patients. Among the cirrhotics, nine (7.5\%) were categorized as Child B or Child C. Twenty-four (20.2\%) had a Model For End-Stage Liver Disease (MELD) score of $\geqslant 10$ and out of these 16 had a score of 15 or more. The anticipated clinical outcomes and risk of decompensation were evaluated using the Baseline-eventanticipation (BEA) score. Eight (6.7\%) patients were categorized as BEA class A (mild risk of decompensation), 105 (88.2\%) as BEA class B (moderate risk), and six (5.0\%) as BEA class C (severe risk). The assessment of disease severity in this cohort is given in Table 2. 


\section{Cureus}

\section{Characteristic}

Splenomegaly

AST to Platelet Ratio Index (APRI) APRI > 1.0

Cirrhosis of liver

Child class of cirrhosis $(n=45)$

A

B

Decompensated disease

Ascites

Hepatic encephalopathy

Median MELD score in cirrhotic patients

MELD $\geq 10$

MELD $\geq 15$

Baseline-event-anticipation (BEA) score (all patients = 119)

BEA-A

BEA-B

BEA-C
Number of Patients (\%)

25 (21\%)

$0.8(0.2-12.2) 49$ (41.2\%)

$45(37.8 \%)$

$36(80 \%)$

$7(15.6 \%)$

$2(4.4 \%)$

$5(4.2 \%)$

$4(3.4 \%)$

$1(0.8 \%)$

$8(6-27)$

$24(20.2 \%)$

$16(13.4 \%)$

$2(1-6)$

$8(6.7 \%)$

$105(88.2 \%)$

$6(5.0 \%)$

\section{TABLE 2: Assessment of disease severity in young hepatitis D virus (HDV) infected patients}

Values are median (range) or number (percentage).

MELD: Model For End-Stage Liver Disease

A comparison of patients with and without cirrhosis was done. Key predictive parameters in patients with cirrhosis were splenomegaly, low total leucocyte count and low platelets, higher bilirubin, aspartate aminotransferase, gamma-glutamyl transferase and international normalization ratio (INR), low albumin, higher AST to platelet ratio index (APRI), and a higher BEA class (Table 3). 


\section{Cureus}

\begin{tabular}{|c|c|c|c|}
\hline Characteristic & Cirrhosis $(n=45)$ & Without cirrhosis $(n=74)$ & p-value \\
\hline Male gender & $40(89 \%)$ & $65(88 \%)$ & 1.000 \\
\hline Age & $21.69 \pm 2.72$ & $21.47 \pm 2.80$ & 0.754 \\
\hline Duration of hepatitis B & $64.13 \pm 60.64$ & $48.70 \pm 52.86$ & 0.167 \\
\hline Duration of hepatitis D & $34.16 \pm 44.09$ & $23.38 \pm 26.93$ & 0.438 \\
\hline Body mass index & $20.88 \pm 3.30$ & $21.80 \pm 3.97$ & 0.191 \\
\hline Splenomegaly & $24(53 \%)$ & $1(1.3 \%)$ & $<0.001^{*}$ \\
\hline Spleen Size & $13.46 \pm 2.89$ & $10.81 \pm 0.95$ & $0.001^{*}$ \\
\hline Previous treatment & $23(51 \%)$ & 35 (47\%) & 0.686 \\
\hline Hemoglobin (g/dl) & $13.36 \pm 1.91$ & $13.76 \pm 1.55$ & 0.375 \\
\hline Total leucocyte count (TLC): (109/L) & $5.55 \pm 2.31$ & $7.04 \pm 2.47$ & $<0.001^{*}$ \\
\hline Platelets count: (109/L) & $120.80 \pm 62.98$ & $227.73 \pm 70.76$ & $<0.001^{*}$ \\
\hline HDV RNA quantitative: $(\log 10 \mathrm{IU} / \mathrm{ml})(\mathrm{n}=97)$ & $5.55 \pm 1.57(n=37)$ & $5.80 \pm 1.56(n=60)$ & 0.501 \\
\hline HBV DNA detected & $27(56 \%)$ & $56(76 \%)$ & 0.071 \\
\hline HBV DNA quantitative: $(\log 10 \mathrm{IU} / \mathrm{ml})(\mathrm{n}=73)$ & $2.96 \pm 1.68(n=25)$ & $3.48 \pm 2.00(n=48)$ & 0.309 \\
\hline HBeAg & $7(15 \%)$ & $13(18 \%)$ & 0.776 \\
\hline Bilirubin (mg/dL) & $1.48 \pm 2.11$ & $0.76 \pm 0.45$ & $0.038^{*}$ \\
\hline Alanine aminotransferase (ALT) (IU/L) & $102.07 \pm 99.54$ & $105.80 \pm 100.81$ & 0.417 \\
\hline Aspartate aminotransferase (ASI) (IU/L) & $96.18 \pm 92.95$ & $73.69 \pm 68.89$ & $0.035^{n}$ \\
\hline Gamma glutamyl transferase (IU/L) & $104.13 \pm 159.10$ & $56.27 \pm 66.51$ & $0.008^{*}$ \\
\hline Alkaline phosphatase (IU/L) & $138.71 \pm 68.40$ & $132.41 \pm 71.98$ & 0.182 \\
\hline Albumin (g/dL) & $3.77 \pm 0.59$ & $4.23 \pm 0.33$ & $<0.001^{x}$ \\
\hline INR & $1.2538 \pm 0.37$ & $1.05 \pm 0.13$ & $0.005^{*}$ \\
\hline Creatinine (mg/dL) & $0.85 \pm 0.25$ & $0.81 \pm 0.15$ & 0.650 \\
\hline Sodium (mmol/L) & $136.71 \pm 5.38$ & $138.93 \pm 2.20$ & 0.098 \\
\hline AST to Platelet Ratio Index (APRI) & $2.51 \pm 2.22$ & $0.88 \pm 0.92$ & $<0.001^{*}$ \\
\hline \multicolumn{4}{|l|}{ Baseline-event-anticipation (BEA) score } \\
\hline BEA-A & $0(0 \%)$ & $8(11 \%)$ & $0.001^{*}$ \\
\hline BEA-B & $39(8 / \%)$ & $66(89 \%)$ & \\
\hline BEA-C & $6(13 \%)$ & $0(0 \%)$ & \\
\hline
\end{tabular}

\section{TABLE 3: Comparison of hepatitis D patients with and without cirrhosis}

Values are mean \pm standard deviation or number $(\%)$

* Significant $p$-values by independent sample Mann-Whitney U test

HDV: Hepatitis D virus; HBV: Hepatitis B virus; HBeAg: Hepatitis B e-antigen; INR: International normalization ratio.

Linear regression analysis by stepwise method revealed that splenic size $(\mathrm{cm})$, platelet count, and albumin levels were independently associated with cirrhosis with p-values of $<0.001,<0.001$, and $<0.003$ and Beta coefficients of $0.391,-0.356$, and -0.221 , respectively. The areas under the ROC curves for these variables were $0.768,0.878$, and 0.786 , respectively (Figure 1 ). 


\section{Cureus}
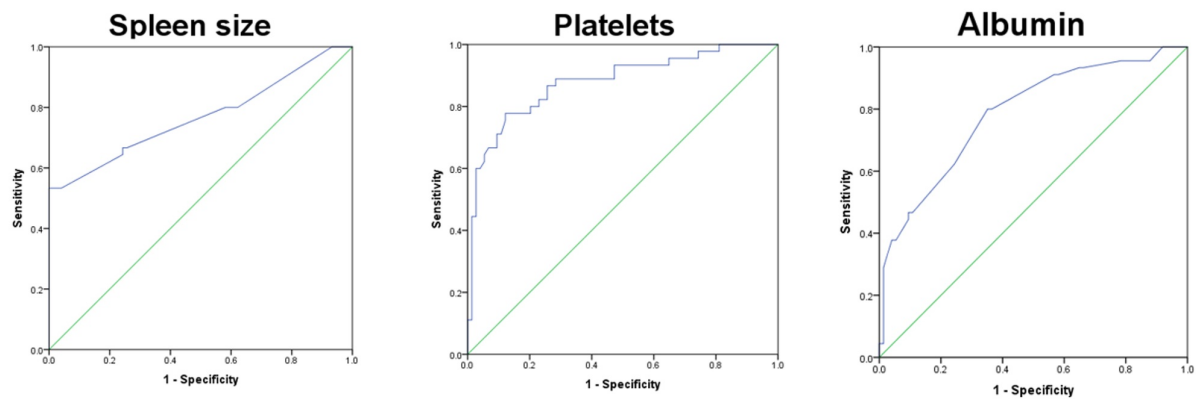

FIGURE 1: The association of spleen size $(\mathrm{cm})$, platelet count, and albumin levels with cirrhosis. The areas under the ROC curves for these variables are $0.768,0.878,0.786$, respectively.

ROC: Receiver operating characteristic

Binomial logistic regression with all three variables (splenic size, platelet count, and albumin levels) or with splenic size and platelet counts alone was also able to accurately predict cirrhosis. Both models were significantly better than the null model (Chi-Square Test, $\mathrm{p}<0.05$ ). The splenic size and platelet count contributed to most of the accuracy of the model. The equations used to calculate log-odds for cirrhosis were derived from this analysis and are given below for each model:

3 variable model

$\ln (\mathrm{P} 0 \mathrm{c} / 1-\mathrm{P} 0 \mathrm{c})=0.842 *($ Splenic Size in $\mathrm{cm})-1.482 *($ Albumin $)-0.023 *($ Platelet count $)-0.324$

2 variable model

$\ln (\mathrm{P} 0 \mathrm{c} / 1-\mathrm{P} 0 \mathrm{c})=0.828 *($ Splenic Size in $\mathrm{cm})-0.027 *($ Platelet count $)-5.094$

Where P0cis is the probability of cirrhosis and $\ln$ is the natural log.

Splenic size is in cm and platelet count in xyz x 109/L

The right-hand side of the equation can be independently considered as a new scoring system. ROC curves have constructed using the results from each model (Figure 2).
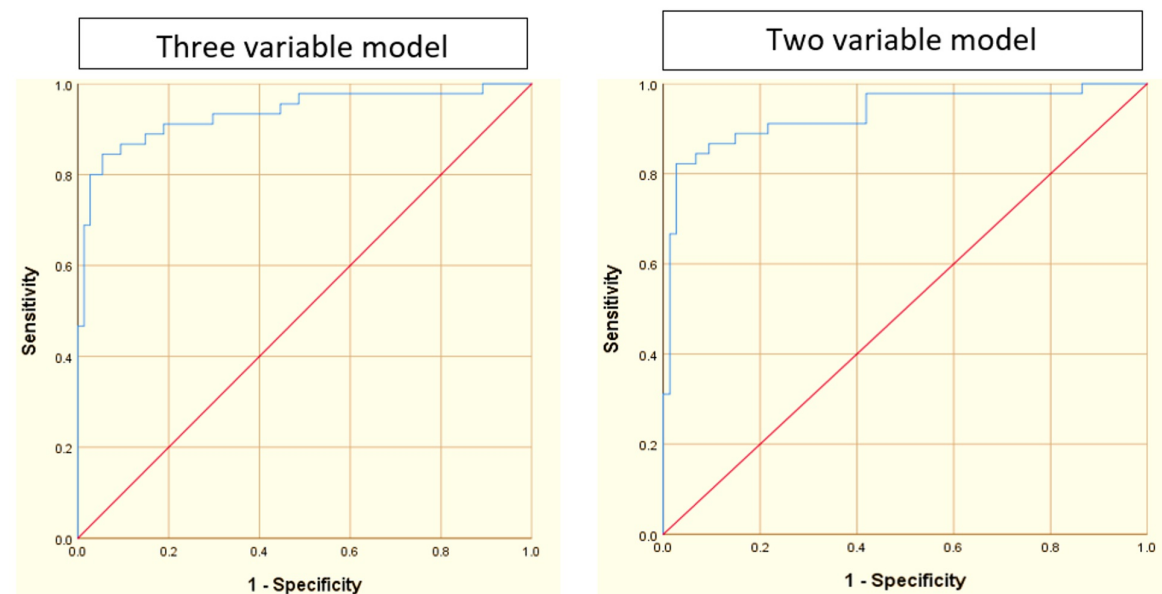

FIGURE 2: Results of binomial Logistic Regression. ROC curves for the three-variable and two-variable models. The areas under the ROC curves for these models are 0.935 and 0.932 , respectively.

ROC: Receiver operating characteristic 
The area under the receiver operating characteristics (AUROC) was 0.935 for the three-variable model and 0.932 for the two-variable model. The cut-off for the prediction of cirrhosis, for both models, can be taken as a value between -0.55 and +0.55 of the right-hand side of the equation, i.e., values greater than +0.55 highly predictive of cirrhosis and values less than -0.55 less likely to be cirrhosis. This has the highest combination of sensitivity and specificity (1.795).

Finally, patients who had previously received pegylated interferon therapy, displayed lower bilirubin $(0.90 \pm$ 0.97 vs $1.16 \pm 1.68$ ), lower alanine aminotransferase (ALT) $85.0 \pm 54$ vs $122.7 \pm 12$, lower aspartate aminotransferase (AST) $(65.6 \pm 40$ vs $97.9 \pm 101)$, and lower APRI $(1.17 \pm 1.16$ vs $1.81 \pm 2.10)$ when compared with patients without treatment, despite having a longer duration of disease (in months since HBV diagnosis: $72.14 \pm 56.66$ vs $37.80 \pm 50.73$ and HDV diagnosis $37.98 \pm 36.63$ vs $17.44 \pm 29.64)$, $(\mathrm{p}<0.05$ each).

\section{Discussion}

Hepatitis D, driven by genotype $1 \mathrm{HDV}$ is a growing burden in Pakistan [12,13]. Various studies from across the country have shown a prevalence of $14-18 \%$ of HDV infection in HBsAg positive individuals $[2,14,15]$. However, the prevalence of anti-HDV antibodies is much higher; up to 37-38\% in HBsAg positive patients with chronic liver disease, visiting liver clinics or hospitals for treatment, seem to harbour the HDV virus $[16,17]$.

The presence of childhood HBV infection appears to be the main risk factor in this population. Chronic HBV infection makes young patients susceptible to the horizontal transmission of HDV in high prevalent areas. HBV/HDV mother-to-child co-transmission is rare $[8,18]$. These findings from Pakistan are broadly similar to those from a study conducted in a remote rural community in central Africa, where a high prevalence of HBsAg carriage and HDV infection in children and adolescents was observed [19]. This is in contrast to the observations from Romania, an Eastern European nation that still has high morbidity from HDV infection, but where the rate of infection has slowed and most patients are more than 50 years of age [20]. Hepatitis Delta International Network (HDIN) data, systematically analyzed by Wranke et al. confirms this disparate observation [21]. On average, hepatitis D patients from South Asia (Pakistan) were younger (32.7 years; range: $11-70$ ) than those from Eastern Europe (35.7 years; range: 1-79). The HDIN data also showed that although HBeAg was negative in the vast majority of hepatitis D cases worldwide (10.3\%-16.1\% were positive), patients from Pakistan were more likely to be HBeAg positive (35.2\%), and HBV DNA positive (60.8\%).

Although it is accepted that the HDV causes a more severe infection at a younger age than HBV monoinfection, studies have not adequately tracked disease progression in the younger population. Indeed, while many studies done in Pakistan have shown that many hepatitis D patients are young adults (21-40 years) [15,22], none have studied the disease exclusively in the 18-25 year demographic.

We previously reported that children with HDV infection have a more aggressive form of liver disease than those with HBV monoinfection [10]. This was observed irrespective of HBeAg status. A comparison of this current study with the previous one done on children shows that male preponderance persists in young adults. However, we accept that this skew may arise from several cultural factors that bias attendance at our clinics. The comparative analysis also showed that the HBV DNA was detectable in about $50 \%$ of children and $70 \%$ of young adults. These patients had low HBV load (median $3.12 \log 10 \mathrm{IU} / \mathrm{ml}$ ), which is expected, given the known suppressive effect of HDV on HBV [13]. On the other hand, HBeAg positivity decreased from $52 \%$ in children to $17 \%$ in the young adults' group. Cirrhosis was present in $37.8 \%$ of young adults versus $27 \%$ of children. It may be that there is an early aggressive phase in children, adolescents, and young adults followed by a more indolent progression. Moreover, there might be two categories of young adults, classified according to the aggressiveness of the disease. The ones with more aggressive disease progressed to cirrhosis at a very rapid pace.

The clinical outcomes associated with HDV infection can be predicted by the baseline-event-anticipation score (BEA score) [11]. The BEA score includes age, sex, the region of origin, bilirubin, platelets, and INR. The problems with employing this scoring system in our study are multifold. In our study cohort, all patients were from Pakistan, hence automatically gained a point. The cohort was below the age of 40 and so a point could not be added. The majority were also male, so they gained one additional point. All these factors limited the usefulness of the BEA score as a predictive tool. Indeed, as a result of these automatic point assignments, most patients fell into the category of moderate risk for poor long-term outcomes. Nonetheless, the scoring system and its classification of the majority is partially vindicated by the presence of cirrhosis in many patients and closely corresponds to the high APRI score in this cohort.

We also propose some parameters in this specific population that can help predict cirrhosis. Previously, the Delta-4 Fibrosis Score was proposed and validated for the accurate prediction of cirrhosis. This score utilized gamma-glutamyl transferase (GGT), ALT, platelet count, and liver stiffness and demonstrated an AUROC of 0.94 in a cohort of 77 HDV positive patients [23]. A similar score, called the Delta Fibrosis score, utilized GGT, age, albumin, and serum cholinesterase, and had an AUROC of 0.87 in a cohort of 100 HDV positive patients [24]. While these scoring systems are very informative, we found that a combination of spleen size and platelet combination is sufficiently sensitive and specific to classify cirrhotics in our demographic. The 
addition of albumin led to only partial gains in overall accuracy. Further testing may be required to validate this new model, but this new scoring system presents as a simplified additional tool, that utilizes noninvasive tests for the prediction of cirrhosis in young adults age 18-25 years with hepatitis D infection.

Finally, it is worth noting that $48.7 \%$ of our patients had received pegylated interferon therapy without sustained response. The lack of response is not surprising. Unlike the South American genotype 3 HDV, the Pakistani genotype $1 \mathrm{HDV}$ does not respond well to pegylated interferon therapy [25-27]. However, in comparison to untreated patients, those who received pegylated interferon had lower bilirubin, transaminases, and a lower APRI. It may be that although sustained suppression of HDV is difficult, there is still some benefit of the treatment in slowing down the process of inflammation and fibrosis.

\section{Conclusions}

In conclusion, our study of 119 hepatitis D patients uncovered key characteristics of this hitherto unstudied cohort. We show that most young adults with hepatitis D of age group 18-25 years in Pakistan are male. About two-thirds of them had HBV DNA detectable but most of them were HBeAg negative. Both factors did not influence the clinical outcome. High values of AST and ALT persisted in this demographic, indicating ongoing inflammatory activity. About one-third of patients had already developed cirrhosis and the rest of the patients were at moderate risk of disease progression. This paints a troubling picture in this demographic. These findings make it incumbent to address the risk factors associated with HDV infection and to implement an effective vaccination program for hepatitis B.

\section{Additional Information}

\section{Disclosures}

Human subjects: Consent was obtained by all participants in this study. Ethics Review Committee Dr. Ziauddin University Hospital issued approval 2040320ZAGE. The Ethics Review Committee reviewed the study protocol and granted access to the data. Our study did not require written/informed consent because of retrospective analysis without disclosing the identity or doing any intervention. The study protocol conforms to the ethical guidelines of the 1975 Declaration of Helsinki as reflected in a priori approval by the institution's human research committee. Animal subjects: All authors have confirmed that this study did not involve animal subjects or tissue. Conflicts of interest: In compliance with the ICMJE uniform disclosure form, all authors declare the following: Payment/services info: All authors have declared that no financial support was received from any organization for the submitted work. Financial relationships: All authors have declared that they have no financial relationships at present or within the previous three years with any organizations that might have an interest in the submitted work. Other relationships: All authors have declared that there are no other relationships or activities that could appear to have influenced the submitted work.

\section{References}

1. Stockdale AJ, Kreuels B, Henrion MYR, et al.: The global prevalence of hepatitis D virus infection: systematic review and meta-analysis. J Hepatol. 2020, 73:523-532. 10.1016/j.jhep.2020.04.008

2. Abbas Z, Jafri W, Raza S: Hepatitis D: scenario in the Asia-Pacific region. World J Gastroenterol. 2010 16:554-562. 10.3748/wjg.v16.i5.554

3. Flodgren E, Bengtsson S, Knutsson M, Strebkova EA, Kidd AH, Alexeyev OA, Kidd-Ljunggren K: Recent high incidence of fulminant hepatitis in Samara, Russia: molecular analysis of prevailing hepatitis B and D virus strains. J Clin Microbiol. 2000, 38:3311-3316. 10.1128/JCM.38.9.3311-3316.2000

4. Rizzetto M, Verme G, Recchia S, et al.: Chronic hepatitis in carriers of hepatitis B surface antigen, with intrahepatic expression of the delta antigen. An active and progressive disease unresponsive to immunosuppressive treatment. Ann Intern Med. 1983, 98:437-441. 10.7326/0003-4819-98-4-437

5. Farci P, Niro GA: Clinical features of hepatitis D. Semin Liver Dis. 2012, 32:228-236. 10.1055/s-0032-1323628

6. Wedemeyer H, Manns MP: Epidemiology, pathogenesis and management of hepatitis D: update and challenges ahead. Nat Rev Gastroenterol Hepatol. 2010, 7:31-40. 10.1038/nrgastro.2009.205

7. Maggiore G, Hadchouel M, Sessa F, et al.: A retrospective study of the role of delta agent infection in children with HBsAg-positive chronic hepatitis. Hepatology. 1985, 5:7-9. 10.1002/hep.1840050103

8. Sellier PO, Maylin S, Brichler S, et al.: Hepatitis B virus-hepatitis D virus mother-to-child co-transmission: a retrospective study in a developed country. Liver Int. 2018, 38:611-618. 10.1111/liv.13556

9. Craxì A, Di Marco V, Volpes R, et al.: Treatment with recombinant alpha 2b-interferon of chronic HDV hepatitis in children. Prog Clin Biol Res. 1991, 364:399-404.

10. Abbas Z, Soomro GB, Hassan SM, Luck NH: Clinical presentation of hepatitis D in Pakistani children . Eur J Gastroenterol Hepatol. 2014, 26:1098-1103. 10.1097/MEG.0000000000000168

11. Calle Serrano B, Großhennig A, Homs M, et al.: Development and evaluation of a baseline-eventanticipation score for hepatitis delta. J Viral Hepat. 2014, 21:154-163. 10.1111/jvh.12251

12. Butt FA, Amin I, Idrees M, Iqbal M: Hepatitis delta virus genotype- 1 alone cocirculates with hepatitis B virus genotypes A and D in Pakistan. Eur J Gastroenterol Hepatol. 2014, 26:319-324. 10.1097/MEG.0000000000000007

13. Moatter T, Abbas Z, Shabir S, Jafri W: Clinical presentation and genotype of hepatitis delta in Karachi . World J Gastroenterol. 2007, 13:2604-2607. 10.3748/wjg.v13.i18.2604

14. Mumtaz K, Hamid SS, Adil S, et al.: Epidemiology and clinical pattern of hepatitis delta virus infection in Pakistan. J Gastroenterol Hepatol. 2005, 20:1503-1507. 10.1111/j.1440-1746.2005.03857.x 
15. Aftab M, Amin I, Idrees M, Ali A, Rafique S, Naz S: Molecular epidemiology of hepatitis delta and hepatitis B viruses circulating in two major provinces (East and North-West) of Pakistan. Infect Genet Evol. 2018, 64:65-69. 10.1016/j.meegid.2018.06.013

16. Amini N, Alavian SM, Kabir A, Aalaei-Andabili SH, Saiedi Hosseini SY, Rizzetto M: Prevalence of hepatitis d in the eastern mediterranean region: systematic review and meta analysis. Hepat Mon. 2013, 13:8210. 10.5812/hepatmon.8210

17. Seetlani NK, Abbas Z, Raza S, Yakoob J, Jafris W: Prevalence of hepatitis D in HBsAg positive patients visiting liver clinics. JPMA J Pak Med Assoc. 2009, 59:434-437.

18. Ranger-Rogez S, Alain S, Denis F: Hepatitis viruses: mother to child transmission (Article in French) . Pathol Biol (Paris). 2002, 50:568-575. 10.1016/s0369-8114(02)00351-6

19. François-Souquière S, Makuwa M, Bisvigou U, Kazanji M: Epidemiological and molecular features of hepatitis B and hepatitis delta virus transmission in a remote rural community in central Africa. Infect Genet Evol. 2016, 39:12-21. 10.1016/j.meegid.2015.12.021

20. Gheorghe L, Csiki IE, Iacob S, et al.: Hepatitis delta virus infection in Romania: prevalence and risk factors . J Gastrointest Liver Dis. 2015, 24:413-421. 10.15403/igld.2014.1121.244.dtv

21. Wranke A, Pinheiro Borzacov LM, Parana R, et al.: Clinical and virological heterogeneity of hepatitis delta in different regions world-wide: the Hepatitis Delta International Network (HDIN). Liver Int. 2018, 38:842-850. 10.1111/liv.13604

22. Khan AU, Waqar M, Akram M, et al.: True prevalence of twin HDV-HBV infection in Pakistan: a molecular approach. Virol J. 2011, 8:420. 10.1186/1743-422X-8-420

23. Da BL, Surana P, Kleiner DE, Heller T, Koh C: The Delta-4 fibrosis score (D4FS): a novel fibrosis score in chronic hepatitis D. Antiviral Res. 2020, 174:104691. 10.1016/j.antiviral.2019.104691

24. Lutterkort GL, Wranke A, Yurdaydin C, et al.: Non-invasive fibrosis score for hepatitis delta . Liver Int. 2017, 37:196-204. 10.1111/liv.13205

25. Borzacov LMP, de Figueiredo Nicolete LD, Souza LFB, Dos Santos AO, Vieira DS, Salcedo JMV: Treatment of hepatitis delta virus genotype 3 infection with peg-interferon and entecavir. Int J Infect Dis. 2016, 46:82-88. 10.1016/j.ijid.2016.03.017

26. Alvarado-Mora MV, Romano CM, Gomes-Gouvêa MS, Gutierrez MF, Carrilho FJ, Pinho JRR: Dynamics of hepatitis D (delta) virus genotype 3 in the Amazon region of South America. Infect Genet Evol. 2011, 11:1462-1468. 10.1016/j.meegid.2011.05.020

27. Abbas Z, Memon MS, Mithani H, Jafri W, Hamid S: Treatment of chronic hepatitis D patients with pegylated interferon: a real-world experience. Antivir Ther. 2014, 19:463-468. 10.3851/IMP2728 